\title{
Prevalência de transtornos ansiosos e algumas comorbidades em idosos: um estudo de base populacional
}

\author{
Prevalence of anxiety disorders and some comorbidities \\ in elderly: a population-based study
}

Mayara B. Machado', Zuleide Maria Ignácio2,3, Luciano K. Jornada², Gislaine Z. Réus², Helena M. Abelaira², Camila 0. Arent², Mágada T. Schwalm4 ${ }^{4}$ Renan A. Ceretta ${ }^{5}$, Luciane B. Ceretta², João Quevedo 2,6,7,8

\section{RESUMO}

Objetivo: Avaliar a prevalência de transtornos ansiosos e fatores associados em uma amostra populacional de idosos do Sul de Santa Catarina. Métodos: Estudo transversal com base em dados populacionais, que avaliou 1.021 indivíduos idosos entre 60 e 79 anos. Foram realizadas entrevistas domiciliares para aferição de variáveis sobre transtornos ansiosos, por meio do questionário MINI, dados sociodemográficos, hipertensão arterial sistêmica (HAS), infarto agudo do miocárdio (IAM) e dosagem de colesterol. Resultados: As prevalências entre os transtornos ansiosos foram de 22,0\% para o transtorno de ansiedade generalizada (TAG); 14,8\% para fobia social (FS); 10,5\% para transtorno do pânico (TP); e 8,5\% para o transtorno obsessivo-compulsivo (TOC). Além disso, 40,5\% dos indivíduos apresentaram pelo menos um transtorno de ansiedade. A distribuição dos transtornos foi semelhante nos dois gêneros; TAG foi mais prevalente nos indivíduos de menor escolaridade; TOC foi mais presente em indivíduos casados ou em união estável. Em relação às variáveis clínicas, HAS foi associada à presença de TOC; FS foi associada com IAM; TOC e FS foram associados com HDL > 40 mg/dL.

\section{Palavras-chave}

Transtornos de ansiedade, doenças cardiovasculares, comorbidades, idosos.
Conclusão: Os dados demonstram que os quadros de ansiedade são muito frequentes em idosos da comunidade, se sobrepõem de forma significativa e estão associados a algumas variáveis clínicas cardiovasculares.

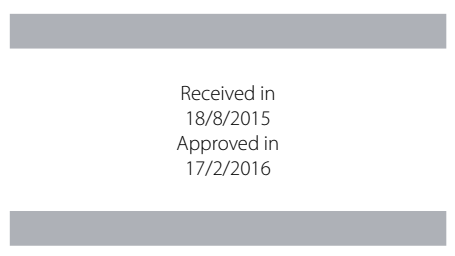

DOI: $10.1590 / 0047-2085000000100$
1 Universidade do Sul de Santa Catarina, Campus Tubarão, Curso de Medicina.

2 Universidade do Extremo Sul Catarinense (Unesc), Unidade Acadêmica de Ciências da Saúde, Laboratório de Neurociências, Programa de Pós-Graduação em Ciências da Saúde.

3 Laboratório de Fisiologia, Farmacologia, Patologia e Psicopatologia, Campus Chapecó, Universidade Federal da Fronteira Sul (UFFS).

4 Universidade do Extremo Sul Catarinense (Unesc), Unidade Acadêmica de Ciências da Saúde, Laboratório de Fisiopatologia Experimental, Programa de Pós-graduação em Ciências da Saúde.

5 Universidade do Extremo Sul Catarinense (Unesc), Unidade Acadêmica de Ciências da Saúde, Laboratório de Microbiologia Experimental, Programa de Pós-graduação em Ciências da Saúde.

6 Translational Psychiatry Program, Department of Psychiatry and Behavioral Sciences, McGovern Medical School, The University of Texas Health Science Center at Houston (UTHealth), Houston, TX, USA.

7 Center of Excellence on Mood Disorders, Department of Psychiatry and Behavioral Sciences, McGovern Medical School, The University of Texas Health Science Center at Houston (UTHealth), Houston, TX, USA.

8 Neuroscience Graduate Program, The University of Texas Graduate School of Biomedical Sciences at Houston, Houston, TX, USA.

Endereço para correspondência: Gislaine Z. Réus

Laboratório de Neurociências,

Programa de Pós-Graduação em Ciências da Saúde,

Unidade Acadêmica de Ciências da Saúde,

Universidade do Extremo Sul Catarinense,

88806-000 - Criciúma, SC, Brasil

Fax: (+55 48) 3431-2736

E-mail: gislainezilli@hotmail.com 


\section{Keywords}

Anxiety disorders, cardiovascular disease, comorbidities, elderly.

\section{ABSTRACT}

Objective: This study evaluated the prevalence of anxiety disorders and associated factors in a population sample of elderly from South of Santa Catarina. Methods: Cross-sectional study based on population data, which evaluated 1,021 elderly individuals, between 60 and 79 years. Home interviews were conducted to measure the variables of anxiety disorders, through of the MINI questionnaire, sociodemographic data, systemic arterial hypertension (SAH), acute myocardial infarction (AMI) and serum cholesterol measurements. Results: The prevalence among anxiety disorders were $22.0 \%$ for generalized anxiety disorder (GAD), $14.8 \%$ for social phobia (FS); 10.5\% for panic disorder (PD); 8.5\% for obsessive-compulsive disorder (OCD), and with only, at least one disorder $40.5 \%$. The distribution of the disorders were similar in both genders, GAD was more prevalent among those with lower education; OCD was more prevalent in individuals who were married or in union stable. In relation to clinical variables, SAH was associated with the presence of OCD; FS was associated with AMl; FS and OCD were associated with HDL $>40 \mathrm{mg} / \mathrm{dL}$. Conclusion: The data demonstrate that anxiety conditions are very common in older adults, significantly overlap and are associated with cardiovascular clinical variables.

\section{INTRODUÇÃO}

Os transtornos de ansiedade são os mais prevalentes entre os transtornos psiquiátricos que acometem a população geral $^{1,2}$. No Brasil, as pesquisas mostram altas prevalências em amostras ambulatoriais, obtendo até taxas de $75 \%$ em tabagistas que procuram tratamento em serviço de psiquiatria ${ }^{3}$.

Em relação ao acometimento dos transtornos de ansiedade entre os gêneros, muitas pesquisas apontam que as mulheres apresentam maior risco de desenvolver transtornos ansiosos ao longo da vida do que os homens ${ }^{4}$ e esses transtornos surgem no início da vida adulta ${ }^{5}$.

Assim como nos adultos, a ansiedade é altamente prevalente entre os idosos, sendo que, da mesma forma que na população geral, é mais proeminente em mulheres e também acomete mais os indivíduos com menor grau de escolaridade e que apresentam outras doenças físicas ${ }^{6,7}$. Os transtornos ansiosos desencadeiam alterações que afetam a qualidade de vida dos idosos, particularmente restringindo suas vidas sociais e diminuindo gradualmente a independência deles ${ }^{8}$. Os sintomas estão geralmente associados a comorbidades psiquiátricas, principalmente a depressão e outras comorbidades clínicas, causando um impacto importante na qualidade de vida desses indivíduos 9 . Os sintomas ansiosos, quando relacionados às comorbidades, estão mais fortemente associados com deficiência física entre os idosos do que nos adultos mais jovens ${ }^{10} \mathrm{e}$ outras situações que limitam ou até incapacitam a vida cotidiana de pessoas idosas ${ }^{11}$. Estudos recentes têm mostrado que é comum em idosos a comorbidade entre transtornos de ansiedade e disfunções cardíacas, respiratórias e do aparelho vestibular, sendo que a ansiedade pode agravar ou ser agravada por outras doenças ${ }^{12}$. Entre as várias COmorbidades clínicas associadas aos transtornos de ansiedade, estão as doenças cardiovasculares, como hipertensão arterial sistêmica (HAS), infarto agudo do miocárdio (IAM) e doença arterial coronariana (DAC) ${ }^{7}$. Os estudos sugerem que os ataques de pânico podem gerar déficits de perfusão miocárdica e pior prognóstico, mesmo em pacientes clinicamente estáveis ${ }^{13}$. Além disso, a ansiedade representa um fator de risco adicional nesses pacientes com DAC e tem se mostrado um fator preditivo de baixa adesão a programas de reabilitação cardíaca e ao tratamento farmacológico ${ }^{13}$.

As projeções indicam que, em 2020, o Brasil contará com um contingente superior a 30 milhões de pessoas acima de 60 anos e será o sexto país do mundo em número de ido$\operatorname{sos}^{14,15}$. Esses indicadores e os dados levantados nas pesquisas acerca dos transtornos psiquiátricos em idosos apontam que é imprescindível mais estudos relacionados aos transtornos ansiosos na população senil, visto que, mesmo tendo uma prevalência expressiva desses transtornos nessa faixa etária, eles continuam sendo subdiagnosticados e subtratados, causando sofrimento e encargos sociais ${ }^{15,16}$. Portanto, além de estudos que possam evidenciar os transtornos de ansiedade e proporcionar diagnósticos mais precisos em idosos, os levantamentos de base populacional podem contribuir de forma significativa para aprimorar os dados estatísticos dessa faixa etária e mostrar outras nuances específicas, subjacentes às populações idosas estudadas, tais como diferenças culturais, e também às características metodológicas de cada estudo6. Portanto, o principal objetivo deste estudo foi avaliar a prevalência de transtornos ansiosos e fatores associados em uma população de idosos do Sul de Santa Catarina, Brasil.

\section{MÉTODOS}

\section{Design e população do estudo}

Este estudo é parte de um projeto piloto, sob o título de Criciúma Diabetes Coorte (CDC), o qual é desenvolvido com a população do município de Criciúma, $\mathrm{SC}^{17}$. O projeto piloto é 
constituído de duas amostras populacionais, uma de diabéticos atendidos no município e outra de indivíduos da comunidade pareados por idade, gênero e região de domicílio. A amostra do presente estudo é constituída pelo extrato de indivíduos da população geral, distribuídos homogeneamente pelas diversas regiões do município, entretanto estratificados pelo pareamento com a população clínica. O estudo foi transversal e foram avaliados 1.021 indivíduos idosos, entre 60 e 79 anos de idade, de uma amostra original de 1.035 (11 se recusaram a participar do estudo e 3 não completaram a entrevista), provenientes da população geral, abrangendo as diferentes regiões do município (amostra não clínica). No presente trabalho, o tamanho amostral foi obtido por conveniência, entretanto se considerarmos que no Censo do IBGE de $2010^{18}$ a população do município era de 14.090 indivíduos na faixa etária do estudo, com nível de confiança de 95\%, podemos considerar o erro amostral de aproximadamente $3 \%$. Os dados foram obtidos em entrevistas domiciliares realizadas por agentes de saúde treinados para a pesquisa.

\section{Diagnósticos psiquiátricos}

Os diagnósticos psiquiátricos foram obtidos a partir de entrevista estruturada na versão em português do Mini International Neuropsychiatric Interview (MINI). O MINI foi validado como um questionário padronizado breve, observando critérios do DSM-IV (APA) e CID-10 (OMS). A padronização do MINI permite sua utilização em pesquisas, podendo ser aplicado em um tempo breve (aproximadamente $15 \mathrm{mi}$ nutos) após um treinamento curto (1 a 3 horas $)^{19}$. A partir do MINI foram avaliados os seguintes transtornos ansiosos: transtorno de ansiedade generalizada (TAG), fobia social (FS), transtorno do pânico (TP) e transtorno obsessivo-compulsivo (TOC).

\section{Características sociodemográficas e clínicas}

Foram avaliadas as seguintes características sociodemográficas: idade, gênero, situação conjugal e grau de escolaridade. Foram também considerados os dados relatados pelos indivíduos acerca de HAS, IAM e acidente vascular encefálico (AVE). Os dados laboratoriais referentes a níveis séricos de colesterol total (CT) e lipoproteínas de alta densidade (High Density Lipoproteins - HDL) foram obtidos em exames coletados para a pesquisa. Essas variáveis foram utilizadas para descrever a população estudada e também foram verificadas as possíveis associações com os transtornos de ansiedade avaliados pelo MINI.

\section{Aspectos éticos}

O planejamento do estudo foi realizado de acordo com a resolução 196/96 do Conselho Nacional de Saúde e aprovado pela Comissão de Ética em Pesquisa - CEP da Universidade do Extremo Sul Catarinense - Unesc, sob protocolo número
310/2009. Após a apresentação e identificação do pesquisador, cada voluntário recebeu informações quanto aos procedimentos, bem como às finalidades do estudo, sua liberdade em aceitar ou não participar e garantia do seu anonimato. Todos os participantes da pesquisa que apresentaram respostas na entrevista, indicando algum transtorno, foram aconselhados a discutir com seus médicos assistentes ou, se não estavam em tratamento, foram encaminhados ao ambulatório de especialidades médicas da Unesc.

\section{Análise estatística}

Após a coleta das informações, os dados foram tabulados e analisados com o programa estatístico Statistical Package for the Social Science (SPSS) versão 16.0. No estudo das associações entre ansiedade com as variáveis sociodemográficas, clínicas e laboratoriais, foram apresentadas as razões de prevalência (prevalência com fator/prevalência sem o fator) e respectivos intervalos de confiança. Para minimizar o risco de viés de confusão no estudo dos fatores sociodemográficos e clínicos associados à presença dos transtornos, foi empregada análise multivariada não hierárquica, por meio de regressão logística binária, controlando entre si, dessa forma, as seguintes variáveis: idade, gênero, situação conjugal, escolaridade, presença de HAS, relato de IAM e níveis de HDL. O estudo da comorbidade entre os transtornos de ansiedade foi analisado com o teste Qui-quadrado, sendo apresentadas as respectivas razões de prevalência e intervalos de confiança. Em todos os testes, o nível de significância adotado foi de 95\% (bilateral).

\section{RESULTADOS}

\section{Diagnósticos psiquiátricos e comorbidades}

Dentre os transtornos de ansiedade, o TAG foi o transtorno mais encontrado na população, com uma prevalência de $22 \%$. Em segundo lugar, foi classificada a FS (14,8\%), seguida por TP $(10,4 \%)$ e TOC (8,5\%). No total da amostra, 413 indivíduos (40,5\%) apresentaram pelo menos um dos quatro transtornos de ansiedade de acordo com os escores do MINI.

Quanto à comorbidade entre os transtornos, dos 413 pacientes que preencheram critérios para um diagnóstico, 122 (29,5\%) tinham pelo menos mais de um transtorno de ansiedade. Na Tabela 1, estão as distribuições das combinações de diagnósticos duplos e sua proporção do total (1.021), não havendo associação somente entre TAG e TOC. Com relação à prevalência de comorbidades psiquiátricas, os seguintes transtornos e percentuais apresentaram associação com outros transtornos ansiosos: TAG (8,1\%), FS (8,0\%), TP (6,3\%) e TOC $(4,8 \%)$. As comorbidades mais frequentes foram de FS $(4,6 \%)$ seguida de TAG e TP $(4,0 \%)$. 
Tabela 1. Presença de comorbidade entre os transtornos ansiosos em 1.021 indivíduos da comunidade

\begin{tabular}{|c|c|c|c|}
\hline Transtorno & Comorbidade & $\mathrm{n}(\%$ do total) & $\mathrm{p}$ \\
\hline \multirow[t]{4}{*}{ Ansiedade generalizada } & Pânico & $41(4,0 \%)^{*}$ & $\mathrm{p}<0,001$ \\
\hline & Fobia social & $47(4,6 \%)^{*}$ & $p=0,004$ \\
\hline & Obsessivo-compulsiva & $21(2,1 \%)$ & $p=0,621$ \\
\hline & Pelo menos uma comorbidade & $83(8,1 \%)^{*}$ & $\mathrm{p}<0,001$ \\
\hline \multirow[t]{4}{*}{ Pânico } & Ansiedade generalizada & $41(4,0 \%)^{*}$ & $\mathrm{p}<0,001$ \\
\hline & Fobia social & $35(3,4 \%)^{*}$ & $\mathrm{p}<0,001$ \\
\hline & Obsessivo-compulsivo & $16(1,6 \%)^{*}$ & $p=0,010$ \\
\hline & Pelo menos uma comorbidade & $64(6,3 \%)^{*}$ & $\mathrm{p}<0,001$ \\
\hline \multirow[t]{4}{*}{ Fobia social } & Ansiedade generalizada & $47(4,6 \%)^{*}$ & $p=0,004$ \\
\hline & Pânico & $35(3,4 \%)^{*}$ & $\mathrm{p}<0,001$ \\
\hline & Obsessivo-compulsiva & $34(3,3 \%)^{*}$ & $\mathrm{p}<0,001$ \\
\hline & Pelo menos uma comorbidade & $82(8,0 \%)^{*}$ & $\mathrm{p}<0,001$ \\
\hline \multirow[t]{4}{*}{ Obsessivo-compulsivo } & Ansiedade generalizada & $21(2,1 \%)$ & $p=0,621$ \\
\hline & Transtorno de pânico & $16(1,6 \%)^{*}$ & $p=0,010$ \\
\hline & Fobia social & $34(3,3 \%)^{*}$ & $\mathrm{p}<0,001$ \\
\hline & Pelo menos uma comorbidade & $49(4,8 \%)^{*}$ & $p<0,001$ \\
\hline
\end{tabular}

${ }^{*} p<0,05$ no teste Qui-quadrado.

\section{Características sociodemográficas e clínicas}

Os dados sociodemográficos e clínicos estão sintetizados na Tabela 2.

Tabela 2. Distribuição das características sociodemográficas e clínicas em 1.021 indivíduos da comunidade

\begin{tabular}{lcc}
\hline Variável & Categoria & Valor \\
\hline Idade & Média & 67,3 anos \\
& Desvio-padrão & 4,8 anos \\
Gênero & Masculino & $425(41,6 \%)$ \\
& Feminino & $596(58,4 \%)$ \\
Situação conjugal & Com companheiro & $561(54,9 \%)$ \\
& Sem companheiro & $460(45,1 \%)$ \\
Escolaridade & Até 8 anos & $575(56,3 \%)$ \\
& 9 anos ou mais & $446(43,7 \%)$ \\
HAS & Presente & $318(31) 1 \%$, \\
& Ausente & $703(68,9 \%)$ \\
IAM & Presente & $135(13,2 \%)$ \\
& Ausente & $886(86,8 \%)$ \\
HDL & $<40 \mathrm{mg} / \mathrm{dL}$ & $843(82,6 \%)$ \\
& $\geq 40 \mathrm{mg} / \mathrm{dL}$ & $178(17,4 \%)$ \\
\hline
\end{tabular}

Na população de 1.021 idosos estudados, o predomínio $(58,4 \%)$ foi de indivíduos do sexo feminino. A média de idade encontrada foi de 67 anos com desvio-padrão de cinco anos. Quanto à situação conjugal, o maior percentual (54,9\%) dos pacientes vivia com um companheiro e $56,3 \%$ dos pacientes estudaram até $08^{\circ}$ ano escolar (atualmente denominado ensino fundamental, com duração de 9 anos).

Os dados clínicos mostraram que 31\% referiram apresentar hipertensão e grande parte desses pacientes não sofreu IAM ou AVE. Entretanto, 56,9\% dos indivíduos estudados apresentavam CT elevado ( $\geq 200 \mathrm{mg} / \mathrm{dL}$ ) e $82,6 \%$ apresentavam concentrações plasmáticas diminuídas de $\mathrm{HDL}(<40$ $\mathrm{mg} / \mathrm{dL}$ ), em relação aos limites da normalidade.

\section{Associação entre variáveis sociodemográficas e clínicas com transtornos de ansiedade}

Na Tabela 3, encontra-se o estudo da associação entre as variáveis sociodemográficas e clínicas com os transtornos de ansiedade. A distribuição dos transtornos foi semelhante nos dois grupos etários e em ambos os gêneros para os quatro transtornos estudados. O TOC foi significativamente mais frequente em indivíduos casados ou em união estável ( $p=$ 0,001 ), se apresentando acima de duas vezes mais, em relação aos indivíduos que não tinham companheiro; enquanto o TAG foi mais prevalente entre os indivíduos de menor escolaridade, aproximadamente 1,5 vez em relação à maior escolaridade $(p=0,004)$. Em relação às variáveis clínicas, HAS foi significativamente associada à presença de TOC com razão de prevalência próxima a $2(p=0,001)$, por outro lado o relato de IAM, depois de analisado de forma multivariada, foi associado à menor manifestação de $F S(R P=0,62 ; p=0,036)$, bem como os níveis de HDL baixo foram associados à menor manifestação de $F S(R P=0,67 ; p=0,025)$ e $T O C(R P=0,59$; $p=0,016)$. 
Tabela 3. Associação entre as variáveis sociodemográficas, clínicas e laboratoriais com os transtornos ansiosos, em 1.021 indivíduos da comunidade, sendo descrita razão de prevalência (intervalo de confiança 95\%)

\begin{tabular}{|c|c|c|c|c|}
\hline & Ansiedade generalizada & Fobia social & Pânico & Obsessivo-compulsivo \\
\hline $\begin{array}{l}\text { Idade } \\
\text { Até } 65 / 66 \text { anos ou }+\end{array}$ & $\begin{array}{c}0,85(0,65-1,11) \\
p=0,467\end{array}$ & $\begin{array}{c}0,76(0,54-1,09) \\
p=0,057\end{array}$ & $\begin{array}{c}0,83(0,55-1,26) \\
p=0,400\end{array}$ & $\begin{array}{c}0,78(0,48-1,24) \\
p=0,906\end{array}$ \\
\hline $\begin{array}{l}\text { Gênero } \\
\text { Masculino/feminino }\end{array}$ & $\begin{array}{c}0,99(0,78-1,25) \\
p=0,959\end{array}$ & $\begin{array}{c}0,98(0,72-1,32) \\
p=0,807\end{array}$ & $\begin{array}{c}1,12(0,78-1,61) \\
p=0,566\end{array}$ & $\begin{array}{c}1,04(0,69-1,56) \\
p=0,861\end{array}$ \\
\hline $\begin{array}{l}\text { Cônjuge } \\
\text { Com/sem }\end{array}$ & $\begin{array}{c}0,86(0,68-108) \\
p=0,114\end{array}$ & $\begin{array}{c}1,18(0,87-1,59) \\
p=0,609\end{array}$ & $\begin{array}{c}0,79(0,55-1,13) \\
p=0,152\end{array}$ & $\begin{array}{c}2,28(1,44-3,62)^{*} \\
p=0,001\end{array}$ \\
\hline $\begin{array}{l}\text { Escolaridade } \\
\text { Até } 8 / 9 \text { anos ou }+\end{array}$ & $\begin{array}{c}1,46(1,14-1,87)^{*} \\
p=0,004\end{array}$ & $\begin{array}{c}1,28(0,94-1,74) \\
p=0,178\end{array}$ & $\begin{array}{c}1,14(0,79-1,64) \\
p=0,594\end{array}$ & $\begin{array}{c}1,33(0,88-2,02) \\
p=0,099\end{array}$ \\
\hline $\begin{array}{l}\text { Hipertensão } \\
\text { Sim/não }\end{array}$ & $\begin{array}{c}1,06(0,83-1,36) \\
p=0,421\end{array}$ & $\begin{array}{c}1,20(0,88-1,62) \\
p=0,138\end{array}$ & $\begin{array}{c}1,14(0,78-1,66) \\
p=0,474\end{array}$ & $\begin{array}{c}1,97(1,32-2,94)^{*} \\
\mathrm{p}<0,001\end{array}$ \\
\hline $\begin{array}{l}\text { Infarto } \\
\text { Sim/não }\end{array}$ & $\begin{array}{c}1,25(0,92-1,70) \\
p=0,318\end{array}$ & $\begin{array}{c}0,62(0,36-1,06)^{*} \\
p=0,036\end{array}$ & $\begin{array}{c}1,25(0,77-2,04) \\
p=0,501\end{array}$ & $\begin{array}{c}0,66(0,33-1,34) \\
p=0,218\end{array}$ \\
\hline $\begin{array}{l}\text { HDL } \\
\quad<40 \mathrm{mg} / \mathrm{dL} \\
\quad \geq 40 \mathrm{mg} / \mathrm{dL}\end{array}$ & $\begin{array}{c}1,07(0,78-1,47) \\
p=0,895\end{array}$ & $\begin{array}{c}0,67(0,48-0,95)^{*} \\
p=0,025\end{array}$ & $\begin{array}{c}1,11(0,68-1,81) \\
p=0,802\end{array}$ & $\begin{array}{c}0,59(0,38-0,92)^{*} \\
p=0,016\end{array}$ \\
\hline
\end{tabular}

\section{DISCUSSÃO}

Neste estudo, foi observado que o TAG foi o mais prevalente, seguido por FS, TP e TOC. Dentre as variáveis clínicas, grande parte da população em estudo não referiu IAM, AVE ou HAS. Entretanto, quase a totalidade da amostra possuía valores diminuídos de HDL, em relação aos parâmetros de normalidade.

Apesar de subdiagnosticados, em relação à depressão, estudos epidemiológicos vêm mostrando que os transtornos de ansiedade podem ser mais prevalentes do que os transtornos depressivos em indivíduos idosos ${ }^{20}$. Além de prejudicar a qualidade de vida ${ }^{21}$, alguns estudos vêm mostrando que os transtornos de ansiedade em idosos aumentam os riscos de mortalidade ${ }^{22,23}$ e incapacidade ${ }^{24,25}$. Os transtornos ansiosos são altamente prevalentes na população em geral e alguns estudos vêm mostrando que, entre os indivíduos idosos, vários tipos de transtornos de ansiedade se encontram com alta frequência em algumas populações do mundo 1,6,26,27. Entretanto, existem divergências na literatura quanto à prevalência de todas as classificações de transtornos de ansiedade em idosos, as quais parecem estar associadas aos diferentes instrumentos de diagnóstico, outras questões metodológicas e diferenças culturais ${ }^{6,12}$.

Os dados epidemiológicos da DSM-IVTR ${ }^{28}$, com amostras populacionais de adultos moradores nos Estados Unidos, atribuem prevalências de ansiedade inferiores aos resultados deste estudo. A prevalência de TAG durante a vida é 5\% inferior aos 22\% observados neste estudo. Para a FS, foi reportada uma variação entre 3\% e 13\% da população, o que é ligeiramente inferior aos 14,8\% encontrados nesta pesquisa. Com relação ao TOC durante a vida, as pesquisas apontam uma incidência de aproximadamente 3\%16,29, enquanto em pessoas idosas alguns pesquisadores relataram uma preva- lência de $0,8 \%{ }^{26}$. Neste trabalho, foram observados $8,5 \%$ de prevalência de TOC em idosos. Por fim, foi relatada uma prevalência entre $1 \%$ e $2 \%$ de TP nos Estados Unidos ${ }^{29}$ versus 10,4\% observados neste estudo. No Brasil, um estudo sobre transtornos mentais na cidade de São Paulo também verificou prevalências inferiores às encontradas nesta pesquisa, relatando os seguintes percentuais: TAG $(4,5 \%)$, TP $(1,0 \%)$, FS $(2,0 \%)$ e TOC $(2,4 \%)^{30}$. Embora ainda inferiores aos resultados encontrados em nosso trabalho, estes últimos autores já evidenciaram uma prevalência maior de alguns tipos de transtorno do que os estudos realizados em outras populações, sugerindo que alguns fatores, como diferenças culturais, entre outros, possam estar subjacentes aos resultados. Um estudo com populações idosas de regiões urbanas e rurais de alguns países da América Latina, China e Índia revelou maior prevalência de ansiedade em indivíduos da América Latina, indicando que existem diferenças quanto à manifestação de transtornos ansiosos entre diferentes populações ${ }^{31}$.

Resultados divergentes de alguns estudos indicam que a FS se apresenta com uma grande variabilidade quanto à prevalência na população idosa e que os diferentes resultados podem estar associados aos diferentes instrumentos utilizados nas pesquisas ${ }^{32}$. Não obstante a sugestão de que a baixa prevalência de FS possa estar associada ao estilo de vida ou até mesmo ao amadurecimento da personalidade dos idosos ${ }^{32}$, alguns autores sugerem que a metodologia e a interpretação das pesquisas possam estar subdiagnosticando a incidência de FS na população idosa ${ }^{33,34}$. Em um estudo por meio do MINI, a prevalência de FS foi alta em indivíduos idosos, com maior ênfase na população feminina. Os autores sugerem que procedimentos utilizados por intermédio do MINI possam ter aumentado a sensibilidade da avaliação, tendo em vista que os padrões psicométricos mitigaram a 
exclusão de doenças físicas ${ }^{35}$. Ciliberti et al. ${ }^{36}$ também observaram que o direcionamento das propriedades psicométricas, considerando fatores mais preocupantes para os idosos, pode revelar índices de ansiedade social superiores àqueles observados em instrumentos que avaliam a população mais jovem. Outro fator importante é que o presente estudo limitou a faixa etária idosa (acima de 60 anos) e dividiu em duas faixas com ponto de corte de 65 anos. Portanto, é provável que a redução da FS com o avanço da idade em outros estudos não tenha sido replicada aqui, em função da idade menos avançada. A faixa etária até 70 anos pode estar mais exposta a situações sociais que aumentam a vulnerabilidade à FS, a qual pode diminuir em faixas etárias mais estagnadas em relação aos eventos sociais ${ }^{37}$.

Quanto ao gênero, a amostra foi predominantemente feminina. Contudo, não houve relação significativa entre os transtornos ansiosos estudados. Comparando vários trabaIhos da literatura científica, os resultados deste trabalho não replicam os dados apontados ${ }^{31,37}$. Com exceção ao TOC, os estudos com população jovem adulta mostram que os transtornos de ansiedade são mais frequentes em mulheres ${ }^{38}$. Entretanto, alguns autores também propõem que os estudos acerca da ansiedade social devem observar os fatores que provocam preocupações divergentes entre homens e muIheres, sobretudo na população idosa. Considerando essas diferenças, foi possível observar que a ansiedade social pode acometer homens e mulheres e que os fatores predisponentes à ansiedade social em um determinado gênero podem aumentar a prevalência no respectivo gênero ${ }^{38}$. Portanto, os instrumentos de avaliação para determinadas situações que podem predispor transtornos ansiosos devem considerar as diferentes preocupações entre os gêneros ${ }^{38}$. É importante observar também se em determinadas populações os fatores predisponentes aos transtornos de ansiedade entre homens e mulheres estão contrabalançados, em detrimento de alguns fatores em maior evidência em outras populações, aumentando os riscos para determinado gênero.

Em relação à situação conjugal, não foram encontradas diferenças significativas entre indivíduos vivendo com e sem companhia conjugal. Em uma revisão, Vink et al..$^{39}$ encontraram estudos mostrando associação com depressão, mas não encontraram associação entre ansiedade e situação conjugal. Entretanto, neste estudo, observou-se que o TOC foi mais prevalente entre aqueles que possuem companheiro e não houve relação significativa entre os gêneros em relação a esse transtorno. A escolaridade neste estudo apresentou uma associação negativa com o TAG. Entre os indivíduos com escolaridade até 8 anos (Ensino Fundamental), os quais foram a maioria na amostra coletada, o TAG foi significativamente mais prevalente. Alguns estudos têm mostrado que o nível de escolaridade influencia significativamente a frequência de $T A G$, sendo que a morbidade é menor nos indivíduos que completaram o grau universitário ${ }^{40,41}$. Alguns estudos com idosos verificaram que o baixo nível de educação foi associado positivamente com alguns transtornos de ansiedade e TAG especificamente ${ }^{6,42}$. Em uma ampla revisão, os autores mostraram apenas um trabalho associando o nível de escolaridade com transtornos de ansiedade, enquanto o nível de escolaridade foi amplamente relacionado com depressão em indivíduos idosos. Entretanto, no mesmo estudo os autores observaram índices significativos de comorbidade entre depressão e ansiedade ${ }^{39}$. O aumento da prevalência de ansiedade em indivíduos de baixa escolaridade pode estar associado à baixa independência econômica e às suas dificuldades em encontrar condições que possam amenizar seus problemas 6 .

A associação de transtornos ansiosos com as variáveis clínicas foi significativa em relação ao TOC e FS. A HAS, por exemplo, foi associada à presença de TOC. Os relatos de IAM foram associados à FS. O TOC e FS foram associados a níveis acima de $40 \mathrm{mg} / \mathrm{dL}$ de HDL. Diferentemente do que poderia se esperar, o TAG não esteve associado às comorbidades médicas pesquisadas neste estudo. Alguns pesquisadores vêm mostrando que os transtornos ansiosos, de forma geral, estão associados a uma variedade de comorbidades físicas e, entre elas, estão algumas doenças cardíacas ${ }^{7,43}$. Outros estudos observaram que os transtornos de ansiedade aumentam os riscos de mortalidade por doenças cardiovasculares em pacientes idosos ${ }^{36}$. Quanto aos escores de ansiedade e HAS, alguns trabalhos mostraram uma correlação significativamente positiva em pacientes idosos ${ }^{44,45}$, porém, em outras pesquisas, os autores não observaram uma associação de HAS e transtornos ansiosos ${ }^{43}$. Os dados da literatura, juntamente com os resultados do presente estudo, devem ser observados com cautela, tendo em vista os diversos trabalhos mostrando comorbidades entre doenças cardiovasculares e outras doenças físicas e transtornos de ansiedade. Portanto, em novas pesquisas é importante estudar a relação entre ansiedade e HAS, por meio de protocolos mais focados para essa correlação.

Os níveis de HDL foram correlacionados positivamente com FS e TOC neste estudo. Poucos estudos científicos trazem resultado sobre os níveis de colesterol e transtornos específicos de ansiedade. Um estudo mostrou que o TOC e a TAG foram associados positivamente com aumento de $L D L$ e redução de $H D L{ }^{46}$. Em alguns outros estudos, os pesquisadores reportam que os níveis de colesterol foram altos em vários transtornos de ansiedade, como TAG, TOC e TP47 e $\mathrm{FS}^{48}$. Neste estudo, os níveis de colesterol não foram associados positivamente com transtornos de ansiedade. Os níveis de HDL foram associados positivamente com TOC e FS, um resultado que diverge dos dados encontrados em outras pesquisas. Portanto, é importante a realização de outros estudos, para verificar se esses resultados serão replicados e quais fatores podem estar subjacentes a essa população. 
Quanto à comorbidade entre transtornos ansiosos, os dados chamam a atenção para a gravidade devida à sobreposição de quadros psiquiátricos. Os dados da literatura também destacam as comorbidades psiquiátricas, principalmente envolvendo transtornos depressivos com algum transtorno de ansiedade $^{12}$. Um estudo mostrou que o TP, além de estar associado com depressão, também foi correlacionado positivamente com FS, ratificando o resultado deste estudo ${ }^{49}$. Um outro estudo também com idosos mostrou a prevalência de sintomas depressivos em indivíduos que apresentaram condições associadas à presença de sintomatologia depressiva como percepção negativa da saúde, tabagismo, dentre outros ${ }^{50}$.

As interpretações desses dados devem ser feitas com certo cuidado, visto que os diagnósticos foram aferidos por meio de entrevista estruturada, não sendo o padrão de referência que é a entrevista clínica. Pode se esperar que instrumentos de avaliação tendam a elevar as prevalências. Entretanto, mesmo que a prevalência inclua dados subclínicos, reflete um grau considerável de sofrimento na população idosa da comunidade avaliada.

A amostra deste estudo foi obtida de um grupo extraído do projeto piloto $C D C$, portanto as técnicas de amostragem não foram delineadas especificamente para um estudo de base populacional, podendo incorrer em possíveis vieses de seleção. Entretanto, foram alocados indivíduos das diferentes regiões do município, tratando-se de uma amostra da população geral, o que não caracteriza uma população clínica específica, que é comum nos estudos em nosso meio. Dessa forma, essa metodologia faz dessa população uma amostra que reflete a população geral, sem um risco aumentado de comorbidades das amostras clínicas. Outro aspecto importante a ser considerado foi a constatação de que diversos indivíduos estavam em uso de psicofármacos e outras formas de tratamento para ansiedade, cujos tratamentos apontavam para uma provável ocorrência de pacientes subtratados, não só pela falta de diagnóstico. A aferição dessas taxas com medicamentos e acompanhamentos específicos fugia aos objetivos da pesquisa e, portanto, novos estudos são necessários para compreender melhor a magnitude dos transtornos de ansiedade.

Os dados obtidos da população de idosos neste estudo demonstram que os quadros de ansiedade são muito frequentes. Entretanto, alguns resultados desta pesquisa se mostram com bastante discrepância em relação a outros trabalhos, o que denota um pouco de cautela e indica a necessidade de mais estudos com outras ferramentas de avaliação, para que se possa fazer um comparativo com esses resultados. Embora com alguns resultados discrepantes em relação aos dados encontrados na literatura, referentes a outras populações ou com diferentes metodologias, os resultados deste estudo confirmam a frequência elevada de transtornos ansiosos na população idosa e destacam que esses transtornos se sobrepõem de forma significativa, o que pode refletir em outras comorbidades médicas apontadas nas pesquisas e aumentar o sofrimento desses indivíduos. Outro fator importante é que o percentual de pacientes com depressão maior também foi bastante elevado (dados não mostrados), portanto, os transtornos ansiosos podem estar subjacentes a outros quadros psiquiátricos, o que pode ter elevado a sua prevalência na população estudada. Ao apontar uma frequência elevada de transtornos ansiosos e comorbidades na população de idosos, esses dados destacam a importância de investigações clínicas mais detalhadas nesta população e sinalizam a necessidade de mais estudos populacionais, com objetivos de entender melhor os quadros psiquiátricos, mecanismos biopsicossociais envolvidos e outras características que possam estar subjacentes à população estudada e que permitam uma extrapolação para outras populações idosas.

\section{CONTRIBUIÇÕES INDIVIDUAIS}

Todos os autores contribuíram significativamente com este manuscrito, na concepção e desenho do estudo, na análise e interpretação dos dados, na elaboração do artigo e revisaram criticamente seu conteúdo intelectual e aprovaram esta versão final.

\section{CONFLITOS DE INTERESSE}

Os autores declaram não haver conflitos de interesse a serem declarados.

\section{AGRADECIMENTOS}

O Laboratório de Neurociências (Brasil) é um dos centros do Instituto Nacional de Medicina Molecular (INCT-MM) e um dos membros do Centro de Excelência em Neurociências Aplicadas de Santa Catarina (Nenasc). A pesquisa foi apoiada por auxílios financeiros vindos do Conselho Nacional de Desenvolvimento Científico e Tecnológico (CNPq) (JQ), Fundação de Amparo à Pesquisa do Estado de São Paulo (Fapesp) (JQ); Instituto Cérebro e Mente (JQ) e Unesc (JQ). JQ é um pesquisador CNPq 1A. O Programa de Psiquiatria translacional (EUA) é financiado pelo Departamento de Psiquiatria e Ciências Comportamentais, McGovern Medical School, University of Texas Health Science Center em Houston (UTHealth).

\section{REFERÊNCIAS}

1. Ritchie K, Artero S, Beluche I, Ancelin M-L, Mann A, Dupuy AM, et al. Prevalence of DSMIV psychiatric disorder in the French elderly population. Br J Psychiatry. 2004;184:147-52. 
2. Kessler RC, Berglund P, Demler 0 , Jin R, Merikangas KR, Walters EE. Lifetime prevalence and age-of-onset distributions of DSM-IV disorders in the National Comorbidity Survey Replication. Arch Gen Psychiatry. 2005;62(6):593-602.

3. Munaretti CL, Terra MB. Transtornos de ansiedade: um estudo de prevalência e comorbidade com tabagismo em um ambulatório de psiquiatria. J Bras Psiquiatr. 2007;56(Supl. 2):108-15.

4. Andrade L, Viana MC, Silveira CM. Epidemiologia dos transtornos psiquiátricos na mulher. Rev Psiquiatr Clín. 2006;33(2):43-54.

5. Kinrys $G$, Wygant LE. Transtornos de ansiedade em mulheres: gênero influencia o tratamento. Rev Bras Psiquiatr. 2005;27(Supl. 2):43-50.

6. Kirmizioglu Y, Doğan 0, Kuğu N, Akyüz G. Prevalence of anxiety disorders among elderly people. Int J Geriatr Psychiatry. 2009;24:1026-33.

7. Moser DK, Dracup K, Evangelista LS, Zambroski CH, Lennie TA, Chung ML, et al. Comparison of prevalence of symptoms of depression, anxiety, and hostility in elderly patients with heart failure, myocardial infarction, and a coronary artery bypass graft. Heart Lung. 2010;39(5):378-85

8. Antunes HK, Stella SG, Santos RF, Bueno OF, Mello MT. Depression, anxiety and quality of life scores in seniors after an endurance exercise program. Rev Bras Psiquiatr. 2005;27(Supl. 4):266-71.

9. Charney DS, Reynolds CF 3rd, Lewis L, Lebowitz BD, Sunderland T, Alexopoulos GS. Depression and Bipolar Support Alliance consensus statement on the unmet needs in diagnosis and treatment of mood disorders in late life. Arch Gen Psychiatry. 2003;60:664-72.

10. Lenze Eric J, Wetherell JL. A lifespan view of anxiety disorders. Dialogues Clin Neurosci. 2011;13:381-99.

11. Norton J, Ancelin ML, Stewart R, Berr C, Ritchie K, Carrière I. Anxiety symptoms and disorder predict activity limitations in the elderly. J Affect Disord. 2012;141(2-3):276-85.

12. Wolitzky-Taylor KB, Castriotta N, Lenze EJ, Stanley MA, Craske MG. Anxiety disorders in older adults: a comprehensive review. Depress Anxiety. 2010;27(2):190-211.

13. Sardinha A, Araújo CGS, Silva ACO, Nardi AE. Prevalência de transtornos psiquiátricos e ansiedade relacionada à saúde em coronariopatas participantes de um programa de exercício supervisionado. Rev Psiquiatr Clín. 2011;38(Supl. 2):61-5.

14. Brenes GA, Penninx BWJH, Judd PH, Rockwell E, Sewell DD, Wetherell JL. Anxiety, depression, and disability across the lifespan. Aging Ment Health. 2008;12(Supl. 1):158-63.

15. Veras R. Envelhecimento populacional contemporâneo: demandas, desafios e inovações. Rev Saúde Pública. 2009;43(Supl. 3):548-54.

16. Sanches S, Osório F, Udina M, Rocío MS, Crippa JAS. Associação entre ansiedade e hipermobilidade articular: uma revisão sistemática. Rev Bras Psiquiatr. 2012;34(Supl.1):53-60.

17. Ceretta LB, Réus GZ, Abelaira HM, Jornada LK, Schwalm MT, Hoepers NJ, et al. Increased prevalence of mood disorders and suicidal ideation in type 2 diabetic patients. Acta Diabetol. 2012;49(Suppl 1):S227-34.

18. IBGE. Censo 2010. Disponivel em: http://cidades.ibge.gov.br/xtras/perfil.php?codmun=42046.

19. Amorim P. Mini International Neuropsychiatric Interview (MINI): validação de entrevista breve para os transtornos mentais. Rev Bras Psiquiatr. 2000;22:106-15.

20. Schuurmans J, van Balkom A. Late-life anxiety disorders: a review. Curr Psychiatry Rep. 2011;13(4):267-73.

21. Revicki DA, Brandenburg N, Matza L, Hornbrook MC, Feeny D. Health related quality of life and utilities in primary care patients with generalized anxiety disorder. Qual Life Res. 2008:17:1285-94.

22. Allgulander C, Lavori PW. Causes of death among 936 elderly patients with 'pure' anxiety neurosis in Stockholm County, Sweden, and in patients with depressive neurosis or both diagnoses. Compr Psychiatry. 1993;34:299-302.

23. Van Hout HPJ, Beekman ATF, de Beurs E, Comijs H, van Marwijk H, de Haan M, et al. Anxiety and the risk of death in older men and women. Br J Psychiatry. 2004;185:399-404.

24. Brenes GA, Guralnik JM, Williamson JD, Fried LP, Simpson C, Simonsick EM. The influence of anxiety on the progression of disability. J Am Geriatr Soc. 2005;53(1):34-9.

25. Prina AM, Ferri CP, Guerra M, Brayne C, Prince M. Prevalence of anxiety and its correlates among older adults in Latin America, India and China: cross-cultural study. Br J Psychiatry. 2011;199:485-91.

26. Krasucki C, Howard R, Mann A. The relationship between anxiety disorders and age. Int J Geriatr Psychiatry. 1998;13:79-99.

27. van Zelst WH, de Beurs E, Beekman AT, Deeg DJ, van Dyck R. Prevalence and risk factors of posttraumatic stress disorder in older adults. Psychother Psychosom. 2003;72:333-42.
28. American Psychiatric Association: DSM-IV-TRTM - Manual diagnóstico e estatístico de transtornos mentais. (trad. Cláudia Dornelles). 4.ed. rev. Porto Alegre: Artmed; 2002.

29. Regier DA, Boyd JH, Burke Jr. JD, Rae DS, Myers JK, Kramer M, et al. One month prevalence of mental disorders in the United States: based on five Epidemiologic Catchment Area sites. Arch Gen Psychiatry. 1988:45:977-86.

30. Viana MC, Andrade LH. Prevalence, age and gender distribution and age-of-onset of psychiatric disorders in the Sao Paulo Metropolitan Area, Brazil: results from the Sao Paulo Megacity Mental Health Survey. Rev Bras Psiquiatr. 2012;34:249-60.

31. Prina AM, Ferri CP, Guerra M, Brayne C, Prince M. Prevalence of anxiety and its correlates among older adults in Latin America, India and China: cross-cultural study. Br J Psychiatry. 2011:199(6):485-91.

32. Bryant C, Jackson $H$, Ames D. The prevalence of anxiety in older adults: methodological issues and a review of the literature. J Affect Disord. 2008:109(3):233-50.

33. Kogan JN, Edelstein BA, McKee DR. Assessment of anxiety in older adults: current status. J Anxiety Disord. 2000;14:109-32.

34. Karlsson B, Sigstrçm R, Waern M, Ostling S, Gustafson D, Skoog I. The prognosis and incidence of social phobia in an elderly population. A 5-year follow-up. Acta Psychiatr Scand. 2010;122:4-10.

35. Carrière I, Ryan J, Norton J, Scali J, Stewart R, Ritchie K, et al. Anxiety and mortality risk in community-dwelling elderly people. Br J Psychiatry. 2013;203(3):303-9.

36. Ciliberti C, Gould C, Smith M, Chorney D, Edelstein B. A preliminary investigation of developmentally sensitive items for the assessment of social anxiety in late life. J Anxiety Disord. 2011;25(5):686-9.

37. Chou K-L, Mackenzie CS, Liang K, Sareen J. Three-year incidence and predictors of firstonset of DSM-IV mood, anxiety, and substance use disorders in older adults: results from Wave 2 of the National Epidemiologic Survey on Alcohol and Related Conditions. J Clin Psychiatry. 2011;72:144-55.

38. Kinrys G, Wygant LE. Transtornos de ansiedade em mulheres: gênero influencia 0 tratamento. Rev Bras Psiquiatr. 2005;27(Supl. 2):43-50.

39. Vink D, Aartsen MJ, Schoevers RA. Risk factors for anxiety and depression in the elderly: a review. J Affect Disord. 2008;106(1-2):29-44.

40. Ansseau M, Fischler B, Dierick M, Albert A, Leyman S, Mignon A. Socioeconomic correlates of generalized anxiety disorder and major depression in primary care: the gadis II study (generalized anxiety and depression impact survey II). Depress Anxiety. 2008;25:506-13.

41. Ma X, Xiang YT, Cai ZJ, Lu JY, Li SR, Xiang YQ, et al. Generalized Anxiety Disorder in China: Prevalence, Sociodemographic Correlates, Comorbidity, and Suicide Attempts. Perspect Psychiatr. Care 2009; 45(Supl. 2):119-27.

42. Beekman, AT, de Beurs E, van Balkom AJ, Deeg DJ, van Dyck R, van Tilburg W. Anxiety and depression in later life: co-occurrence and communality of risk factors. Am J Psychiatry. 2000;157:89-95

43. El-Gabalawy R, Mackenzie CS, Shooshtari S, Sareen J. Comorbid physical health conditions and anxiety disorders: a population-based exploration of prevalence and health outcomes among older adults. Gen Hosp Psychiatry. 2011;33(6):556-64.

44. Paterniti S, Alpérovitch A, Ducimetière P, Dealberto MJ, Lépine JP, Bisserbe JC. Anxiety but not depression is associated with elevated blood pressure in a community group of French elderly. Psychosom Med. 1999;61(1):77-83.

45. Mehta KM, Simonsick EM, Penninx BW, Schulz R, Rubin SM, Satterfield S, et al. Prevalence and correlates of anxiety symptoms in well functioning adults: findings from the health aging and body composition study. J Am Geriatr Soc. 2003;51(4):499-504.

46. Peter H, Hand I, Hohagen F, Koenig A, Mindermann 0, Oeder F, et al. Serum cholesterol level comparison: control subjects, anxiety disorder patients, and obsessive-compulsive disorder patients. Can J Psychiatry. 2002;47(6):557-561.

47. Papakostas GI, Ongür D, losifescu DV, Mischoulon D, Fava M. Cholesterol in mood and anxiety disorders: review of the literature and new hypotheses. Eur Neuropsychopharmacol. 2004;14(2):135-42

48. Peter H, Goebel P, Müller S, Hand I. Clinically relevant cholesterol elevation in anxiety disorders: a comparison with normal controls. Int J Behav Med. 1999;6(1):30-9.

49. Corna LM, Cairney J, Herrmann N, Veldhuizen S, McCabe L, Streiner D. Panic disorder in later life: results from a national survey of Canadians. Int Psychogeriatr. 2007;19(6):1084-96.

50. Ramos GCF, Carneiro JA, Barbosa ATF, Mendonça JMG, Caldeira AP. Prevalência de sintomas depressivos e fatores associados em idosos no norte de Minas Gerais: um estudo de base populacional. J Bras Psiquiatr. 2015;64(2):122-31. 